\title{
Liquid media regulated growth of cinnamon nanoparticles: Absorption and emission traits
}

\author{
Ali Aqeel Salim a, Sib Krishna Ghoshal c, Lau Pik Suan a, Noriah Bidin a, Khaidzir Bin Hamzah b, \\ Maisarah Duralim a, Hazri Bakhtiar a, \\ a Laser Center, Institute for Scientific and Industrial Research, Universiti Teknologi Malaysia, Skudai 81310, Johor, Malaysia \\ b Faculty of Chemical and Energy Engineering, Universiti Teknologi Malaysia, 81310 UTM Johor Bahru, Johor, Malaysia \\ c Advanced Optical Materials Research Group, Physics Department, Faculty of Science, Universiti Teknologi Malaysia, 81310 UTM Johor Bahru, \\ Johor, Malaysia \\ *Corresponding author: hazri@utm.my
}

\section{Article history}

Received 3 May 2018

Revised 25 August 2018

Accepted 11 September 2018

Published Online 25 October 2018

\section{Graphical abstract}

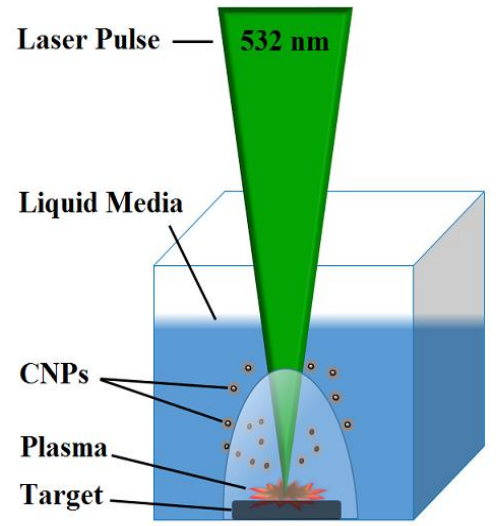

\begin{abstract}
Customized cinnamon-based nanostructures are of great demand for broad arrays of nanomedical, biomedical and photocatalytic purposes. Conventional methods for cinnamon nanomaterials production with desirable optical absorption and emission properties are still limited. This communication elucidates the role of growth media (liquid ethanol and methanol) in controlling the optical qualities of cinnamon nanoparticles (CNPs). Such CNPs of varied morphologies were prepared using pulsed laser ablation in liquid (PLAL) technique (a simple, an inexpensive, and an eco-friendly approach). In the PLAL process, clean cinnamon sticks (as target material purchased from supermarket) were ablated via Q-switched Nd:YAG laser pulse of wavelength $532 \mathrm{~nm}$ operated at optimum fluence of $5.73 \mathrm{~J} / \mathrm{cm}^{2}$. As-synthesized CNPs were characterized at room temperature using ultraviolet-visible (UV-Vis) absorption and photoluminescence (PL) emission spectroscopy. Uniformly dispersed high purity colloidal CNPs with narrow size distribution was achieved. The UV-Vis absorption and the PL spectra of CNPs revealed prominent peaks. These observed distinctive absorption and emission traits of studied CNPs were ascribed to the effects of oxygen deficiency, quantum confinement, crystal defects and existence of cinnamaldehyde compounds. In short, the nucleation and growth of CNPs was significantly sensitive to the chemical character of liquid suspension that provided a favorable thermodynamic condition via entropy driven free energy minimization. It was demonstrated that the structural, morphological, physical, optical properties of such CNPs can be tailored by intuitively selecting the liquid growth media. This disclosure affirmed that the present systematic approach can constitute a basis for the large-scale production of CNPs effective for widespread applications.
\end{abstract}

Keywords: PLAL, CNPs, Liquid media, Absorption, Emission

(c) 2018 Penerbit UTM Press. All rights reserved

\section{INTRODUCTION}

Of late, rapid progress in the field of nanoscience and nanotechnology often relied on tiny organic nanoparticles and nanostructures especially for biomedical and biphotonic applications (Asahi et al., 2008; Islam et al., 2018). A growing interest in the green technology of organic nanomaterials has been triggered due to the possible alteration of the nanomaterials structures and morphologies compared to their bulk counterpart. Organic nanoparticles (ONPs) suspended in solutions are preferred because of their physiochemical structure, size and shape dependent attributes (Salim and Bidin, 2017). These unique features of nanostructures has been contributed in several applications such as solar cell, sensors, bioimaging, biomedicine, etc. to cite a few (Oliveira et al., 2017; Islam et al., 2017). Diverse approaches have been adopted to grow different inorganic nanostructures of iron oxide, silver, copper, gold, etc. However, only few methods have been developed for the synthesis of ONPs (Islam et al., 2017; Rao et al., 2012; An et al., 2002). The common and traditional technique for ONPs synthesis is the re-precipitation and ball milling (Malik et al., 2012). The achieved particle size by these methods is over sub-micrometer. It has been realized that for NPs size below $100 \mathrm{~nm}$ the control of morphologies is yet very difficult, thereby remains a major limitation towards biomedical applications. (Malik et al., 2012; Pileni, 2003; Fernández-Álvarez et al., 2018).

Meanwhile, pulsed laser ablation in liquid (PLAL) technique being a low cost, simple, environmental friendly with large-scale reproducibility of high purity and stable ONPs has been emerged as a handy method to grow NPs (Tsuji et al., 2001; Yan and Chrisey, 2012). PLAL is a variable and eco-friendly method that permits the synthesis of numerous types of nanostructures without requiring any toxic chemicals (Wagener et al., 2010; Salim et al., 2018a). Some recent reports revealed that cinnamon nanoparticles (CNPs) are greatly favorable organic nanomaterial for biomedical applications especially as antibacterial agent (Salim et al., 2017; Salim et al., 2018b).

Since ages, cinnamon has popularly been used as traditional herbal medicine and wide range of purposes in cosmetic, treatment, food ingredients, etc. (Hamidpour et al., 2015). However, its absorption and 
emission traits at nanoscale have not been widely explored. Considering the immense benefits of cinnamon nanostructures, CNPs were synthesized in varied liquid media (ethanol and methanol) at optimum laser fluence of $5.73 \mathrm{~J} / \mathrm{cm}^{2}$ via PLAL technique. Such CNPs disclosed distinct morphologies and optical properties, controllable via laser parameters and growth media. The absorption and emission behavior of as-prepared CNPs were discerned to be sensitive to the nature of liquid media. Results were discussed in terms of various growth mechanisms.

\section{EXPERIMENTAL PROCEDURE}

\section{Synthesis of CNPs}

Analytical grade ethanol $\left(\mathrm{C}_{2} \mathrm{H}_{5} \mathrm{OH}, 96 \%\right.$ purity $)$ and methanol $\left(\mathrm{CH}_{3} \mathrm{OH}, 96 \%\right.$ purity) were purchased from Sigma Aldrich (Malaysia). These liquids medium were used to grow the CNPs at room temperature via PLAL technique. Chinese cinnamon sticks (cassia) were purchased from the local supermarket at Malaysia. Each stick was cut into small pieces and rinsed with sterile water plus acetone solvent. In the PLAL strategy, a Q-switched pulsed Nd:YAG laser (repetition rate of $1 \mathrm{~Hz}$, pulse duration of $8 \mathrm{~ns}$, wavelength of $532 \mathrm{~nm}$, laser fluence of 5.73 $\mathrm{J} / \mathrm{cm}^{2}$ ) was utilized to ablate the bulk cinnamon stick (as target material for CNPs growth). First, the cinnamon stick (target) was immersed at the bottom of beaker filled with $5 \mathrm{ml}$ of liquid media (ethanol and methanol) and then the laser pulse was directly shot on the target surface (at a rate of 1000 pulse/sec) through a focusing lens. The interaction between the energetic laser photon (by $5.73 \mathrm{~J} / \mathrm{cm}^{2}$ of laser fluence) and target surrounded by the liquid media allowed the ablation of cinnamon ultrafine particulates (so called plasma plume) and subsequent nucleation in the form of CNPs. The formed plasma plume on the target surface was cooled down, wherein the liquid media (ethanol and methanol) played a significant role. During ablation, the target was heated through dynamic confinement of liquid media at the surface. The creation of plasma in the medium generated a cavitation bubble which was eventually expanded and then collapsed, driving highly energetic species and producing cinnamon hydroxide materials from the target surface (Salim and Bidin, 2017; Peng et al., 2006; Dimitrakopoulou et al., 2012).

\section{Characterizations of CNPs}

The UV-Vis absorption (PerkinElmer Lambda 25 Spectrometer) and PL emission (Perkin-Elmer Luminescence Spectrophotometer, LS55) spectra of as-grown CNPs existed inside the liquid media (methanol and ethanol) were recorded at room temperature. The absorption of CNPs was measured using a quartz cuvette with a pathlength of $0.5 \mathrm{~cm}$.

\section{RESULTS AND DISCUSSION}

Fig. 1(a) shows the absorption spectra of CNPs prepared in ethanol and methanol liquid media (at optimum laser fluence of $5.73 \mathrm{~J} / \mathrm{cm}^{2}$ ). The absorption peak positions in the wavelength range have been slightly altered from $321 \mathrm{~nm}$ for ethanol and $325 \mathrm{~nm}$ for methanol as well as the intensities of CNPs, indicating that the difference in their growth media were dependent nanomorphologies (size and shape). Where the size of CNPs grown inside ethanol obtained smaller and monodispersed when compared with CNPs obtained inside methanol. It verified that the structure and morphology of CNPs were decided by the nature of liquid media and laser parameters. Besides, the variation in the solution colour (Inset of Fig. 1(a)) indeed confirmed the evolution in the CNPs morphologies and optical properties. The growth of highly crystalline CNPs with spherical (in ethanol) and elliptical (in methanol) shapes has been reported (Salim et al., 2017; Salim et al., 2018b) where the NPs of smaller dimensions were found to influence the absorption intensity through their higher surface damping. Furthermore, the full width at half-maximum (FWHM) under the absorption peaks of CNPs were estimated to be $43.15 \mathrm{~nm}$ (in ethanol) and $52.26 \mathrm{~nm}$ (methanol), respectively (Fig. 1(b)). This increasing trend of FWHM was mainly attributed to the decrease of CNPs size, enhanced surface damping and media oxidation (Coronado et al., 2003; Kumar et al., 2016).
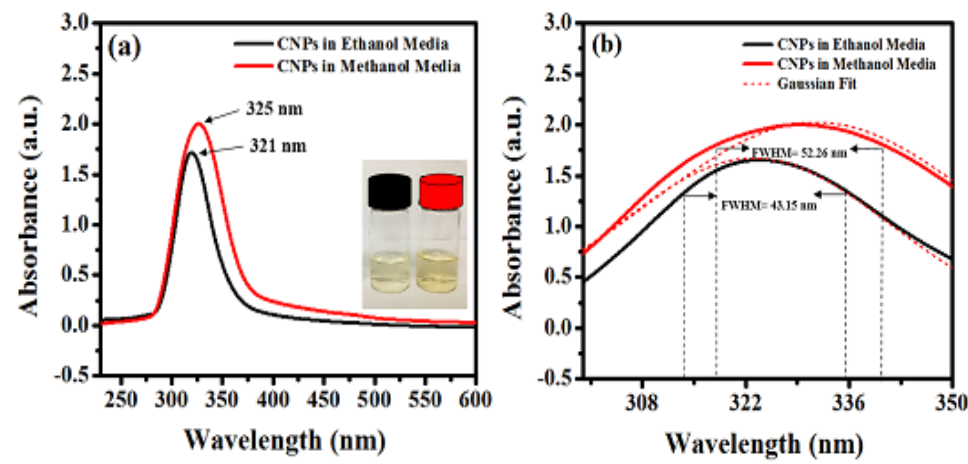

Fig. 1 (a) UV-Vis absorption spectra of prepared CNPs (Inset: colour change of CNPs grown in ethanol as black cap and methanol as red cap, and (b) corresponding FWHM.

Fig. 2 illustrates the PL emission spectra (at the same excitation wavelength of $350 \mathrm{~nm}$ ) of the as-synthesized CNPs grown in ethanol and methanol growth media. The luminescent properties of CNPs were sensitive to the type of liquid media as confirmed from the PL peak position difference and intensity variation. The CNPs grown in ethanol media displayed the highest intensity accompanied by a blue shift compared to the one grown in liquid methanol. This observation was ascribes to the formation of spherical nature of tiny crystalline CNPs (Salim et al., 2018). CNPs grown in methanol liquid media revealed lower PL peak intensity and peak position at a higher wavelength (431 $\mathrm{nm}$ ) than the one grown in ethanol. This verified the sensitivity of NPs morphology on the methanol oxidation and formation of large number of luminescent canters by CNPs (Salim et al., 2017). The strong emission from CNPs grown in both liquid media was due to the enhanced absorption (Salim et al., 2017; Salim et al., 2018a; Coronado et al., 2003).

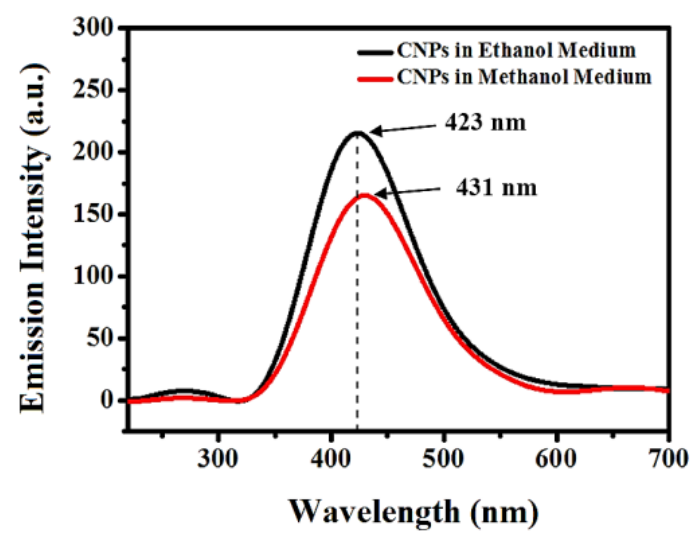

Fig. 2 PL emission spectra of CNPs grown inside ethanol and methanol.

\section{CONCLUSION}

Crystalline, stable and pure CNPs were prepared in ethanol and methanol media using PLAL technique. The influence of growth medium on the optical absorption and PL emission properties of assynthesized CNPs was determined. The revelation of UV-Vis absorption peaks at $321 \mathrm{~nm}$ (ethanol) and $325 \mathrm{~nm}$ (methanol) confirmed the nucleation of CNPs. Meanwhile, the broadening and shift in the absorption peaks of CNPs grown in ethanol and methanol media were attributed to the quantum size effects. The observed prominent PL emission peaks of CNPs at $423 \mathrm{~nm}$ (ethanol) and $431 \mathrm{~nm}$ (methanol) supported their media dependent structure and morphology evolution. The achieved unique optical features of proposed CNPs were attributed to the presence of cinnamaldehyde compounds, crystal defects, oxygen deficiency, and quantum confinement effect. PLAL technique for the CNPs production displayed many advantages such as scaled-up productivity, effortlessness, eco-friendliness, cost-effectiveness and accuracy. It was demonstrated that the morphology and optical. 
characteristics of CNPs can be customized by adjusting the nature of liquid media and laser parameters. In short, these green CNPs with desirable optical traits could be advantageous for biphotonic and biomedicine applications.

\section{ACKNOWLEDGEMENT}

This work was financially supported by the Universiti Teknologi Malaysia under the Research University Grant and Ministry of Higher Education Malaysia GUP/RU/KPT 4L671, 18H68, 17H19 and 18H90.

\section{REFERENCES}

An, B. K., Kwon, S. K., Jung, S. D., Park, S. Y. (2002). Enhanced emission and its switching in fluorescent organic nanoparticles. Journal of the American Chemical Society, 124(48), 14410-14415.

Asahi, T., Sugiyama, T., Masuhara, H. (2008). Laser fabrication and spectroscopy of organic nanoparticles. Accounts of Chemical Research, 41(12), 1790-1798.

Coronado, J. M., Kataoka, S., Tejedor-Tejedor, I., Anderson, M. A. (2003). Dynamic phenomena during the photocatalytic oxidation of ethanol and acetone over nanocrystalline TiO2: Simultaneous FTIR analysis of gas and surface species. Journal of Catalysis, 219(1), 219-230.

Dimitrakopoulou, M., Gorantla, S., Thomas, J., Gemming, T., Cuniberti, G., Büchner, B., Rümmeli, M. H. (2011). Understanding the growth of amorphous $\mathrm{SiO} 2$ nanofibers and crystalline binary nanoparticles produced by laser ablation. Nanotechnology, 23(3), 035601.

Fernández-Álvarez, M., Velasco, F., Bautista, A. (2018). Effect on wear resistance of nanoparticles addition to a powder polyester coating through ball milling. Journal of Coatings Technology and Research, 15(4), 771779 .

Hamidpour, R., Hamidpour, M., Hamidpour, S., Shahlari, M. (2015). Cinnamon from the selection of traditional applications to its novel effects on the inhibition of angiogenesis in cancer cells and prevention of Alzheimer's disease, and a series of functions such as antioxidant, anticholesterol, antidiabetes, antibacterial, antifungal, nematicidal, acaracidal, and repellent activities. Journal of Traditional and Complementary Medicine, 5(2), 6670 .

Islam, S., Bidin, N., Osman, S. S., Krishnan, G., Salim, A. A., Riaz, S., Sanagi, M. M. (2017). Synthesis and characterization of Ni NPs-doped silica-titania nanocomposites: structural, optical and photocatalytic properties. Applied Physics A, 123(1), 67.
Kumar, A., Prasad, M., Shail, S., Baghra, C., Mohanty, B. N., Bhatt, R. B., Kar, R. (2016). Synthesis of uranium-di-oxide nanoparticles by pulsed laser ablation in ethanol and their characterisation. Nano-Structures \& NanoObjects, 7, 75-80.

Malik, M. A., Wani, M. Y., Hashim, M. A. (2012). Microemulsion method: a novel route to synthesize organic and inorganic nanomaterials: 1st nano update. Arabian Journal of Chemistry, 5(4), 397-417.

Oliveira, C. A., Volanti, D. P., Nogueira, A. E., Zamperini, C. A., Vergani, C. E., Longo, E. (2017). Well-designed $\beta$-Ag2MoO4 crystals with photocatalytic and antibacterial activity. Materials \& Design, 115, 73-81.

Peng, Z., Spliethoff, B., Tesche, B., Walther, T., Kleinermanns, K. 2006. Laserassisted synthesis of Au-Ag alloy nanoparticles in solution. The Journal of Physical Chemistry. B 110, 2549-54.

Pileni, M. P. (2003). Nanocrystals: Fabrication, organization and collective properties. Comptes Rendus Chimie, 6(8-10), 965-978.

Rao, C. N. R., Matte, H. R., Voggu, R., Govindaraj, A. (2012). Recent progress in the synthesis of inorganic nanoparticles. Dalton Transactions, 41(17), 5089-5120.

Salim, A. A., Bidin, N., Ghoshal, S. K., Islam, S., Bakhtiar, H. (2018a). Synthesis of truncated tetrahedral cinnamon nanoparticles in citric acid media via PLAL technique. Materials Letters, 217, 267-270.

Salim, A. A., Bidin, N. (2017). Pulse Q-switched Nd: YAG laser ablation grown cinnamon nanomorphologies: Influence of different liquid medium. Journal of Molecular Structure, 1149, 694-700.

Salim, A. A., Bidin, N., Ghoshal, S. K. (2018b). Growth and characterization of spherical cinnamon nanoparticles: Evaluation of antibacterial efficacy. $L W T, 90,346-353$.

Salim, A. A., Bidin, N., Lafi, A. S., Huyop, F. Z. (2017). Antibacterial activity of PLAL synthesized nanocinnamon. Materials \& Design, 132, 486-495.

Tsuji, T., Iryo, K., Nishimura, Y., Tsuji, M. (2001). Preparation of metal colloids by a laser ablation technique in solution: influence of laser wavelength on the ablation efficiency (II). Journal of Photochemistry and Photobiology A. Chemistry, 145(3), 201-207.

Wagener, P., Schwenke, A., Chichkov, B. N., Barcikowski, S. (2010). Pulsed laser ablation of zinc in Tetrahydrofuran: bypassing the cavitation bubble. The Journal of Physical Chemistry C, 114(17), 7618-7625.

Yan, Z., Chrisey, D. B. (2012). Pulsed laser ablation in liquid for micronanostructure generation. Journal of Photochemistry and Photobiology C: Photochemistry Reviews, 13(3), 204-223. 